\title{
Discussion on Key Points of Ecological Landscape Design in Downtown Landscape Planning
}

\author{
Ruoyao Wang \\ Henan Institute of Education, Zhengzhou, 450046, China
}

\begin{abstract}
Keywords: landscape planning; ecological landscape design; green design; landscaping technology
\end{abstract}

\begin{abstract}
The ecological landscape design is a comprehensive subject integrated with sociology, science, art, and aesthetics. With continuously quickening of construction speed of urban environment, people propose higher requirements for living quality of urban environment. The green ecological environment, low-carbon economy, and other concepts have been rooted in people's awareness, which makes ecological landscape in urban landscape attract great attention. The landscape is scarce greenbelt in the city; in urban construction of ecological environment, in order to promote the sustainable development of landscape planning, the landscape architecture warms up continuously; it not only applies landscaping technology to improve cultural level of landscape, but also adds humanity element to make landscape serve as a place for urban residents' entertainment and promote harmony between human and nature. This paper makes a discussion on key points of ecological landscape design in downtown landscape planning. In urban landscape planning, the ecological landscape design not only covers landscaping design, but also includes green design concept, thus it is a reflection of sustainable development of urban ecological environment. From ancient times to the present, the landscape planning design continues as Chinese traditional culture. Except for a bright scenery line in the city, the excellent landscape planning starts from natural ecosystem under the condition of not damaging urban ecological environment to improve urban environment and improve harmony between human and nature so that the urban landscape can become a tranquil place in noisy city and people can relax themselves here.
\end{abstract}

\section{Connotation of ecological landscape design}

From perspective of ecology and aesthetics, the garden design mainly refers to a reasonable configuration of landscape structure and ecological layout in gardens; in the design, we shall not only consider spatial artistic aesthetics, but also consider the shaping of visual landscape. The landscape design can't be separated from architectural design element and planning design element, and the main purpose is to promote integration of garden architecture and natural landscape, thus it is required to make an overall planning on natural and artificial elements in gardens and take the sustainable development of ecological environment as concept to improve overall harmony of gardens and obtain artistic value.

From perspective of meeting people's spiritual life, the ecological landscape design not only can provide urban people with comfortable ecological environment, but also can let people who come here appreciate picturesque scenery and make this place become a healthy and civilized recreation place. It can be seen that the urban landscape planning is not only a welfare work which can reflect the actual scenery in real life, but also can meet people's material demand such as rest and recreation as a kind of spatial art which reflects social ideology.

\section{Ecological landscape design concept in landscape planning}

With continuous improvement of modern urban residents' mental culture, their understanding of inner connotation of landscape architecture is not limited to a place of rest, recreation, and entertainment, but a place of relaxing themselves. While people step into gardens, appreciate beautiful scenery of gardens and breath the fresh air of gardens, their soul is also purified. Then, we shall inject the ecological concept into landscape design so that the green plants in gardens not only 
can purify the air, but also can adjust air humidity and reduce urban noise.

In garden design, it is required to avoid visualized engineering and blind pursuit for garden scale, but carry out landscape planning and design from perspective of natural ecology so as to make urban landscape architecture conform to ecological concept and blend into natural environment. Because the green plants are planning subject in the shaping of natural landscape, we shall consider local climate environment and cultural features in ecological landscape design. There are suitable green plants under different climate environment. In the process of landscape planting, we shall mainly plant local plants; except for attention paid to variety of plants, we shall also make selection according to climatic environment features; besides, in plant landscape design, we shall pay attention to showing the features of plants of different season so that the gardens can show unique charm of landscape all the year round. However, in construction process of landscape architecture, it is not allowed to damage local ecological environment or configure green plants at will; while ensuring sustainable and stable urban landscape, we shall also ensure diversified growth trend of plants in the garden. Besides, the planning design shall be made according to cultural features of local area to avoid simple imitation so that the landscape architecture can easily blend into whole urban planning.

\section{Main principles for ecological landscape design in landscape planning}

(I) The ecological landscape design in landscape planning shall follow the ecology principle.

In garden design, to maintain ecological balance of gardens is the problem we shall give primary consideration, and the purpose is not only to promote stable growth of green plants in gardens, but also to realize reasonable allocation of plants to promote landscape benefits and ecological benefits of gardens. Therefore, we shall consider the ecological environment and biological characteristics in gardens to realize a scientific plant allocation and reasonable structure planning. According to features of plants, we can plant the symbiotic plants together to exert principle of symbiosis and reciprocity. As for plants for which it is not suitable to plant them together, we shall plant them separately to avoid vicious competition between plants so as to better exert functions of garden plants and keep stability of plant community.

(II) The ecological landscape design in landscape planning shall follow the diversity principle.

In ecological landscape design in landscape planning, the special attention shall be paid to protection on original vegetation in gardens, and the new species are introduced under fixed ecological environment of gardens so as to avoid damage on original species due to simple technological design in landscape design. It can be seen that we shall not only consider local natural ecological environment, but also make research on biological species in garden design. For example, in landscape planting, as for selection of local plants, we mainly make considerations from perspective of high rate of survival; due to the fact that there exists selectivity of plants for survival environment, the selected local plants shall blend in landscaping green thoughts both in climate adaptation and in soil and water requirements. Besides, the landscape architecture is also the sign of urban civilization; in order to make it keep persistent local smell, we shall carry out different distribution on local species of plants.

(III) The ecological landscape design in landscape planning shall follow the principle of adjusting measures to local conditions.

Each kind of plant has its own growth characteristics and also selects its survival environment. In ecological landscape design, we shall fully master features of selected plants, and suitably plant the plants according to local climatic environment and soil condition so as to improve survival rate of plants. In garden planning, the features of ecological community in ecological landscape are mainly reflected via a way of manual technique. As a landscape design with relative independence in landscape architecture, the ecological landscape design is of important significance for sustainable plant landscaping.

(IV) The ecological landscape design in landscape planning shall follow the principle of reasonable arrangement.

The landscape planning is an important part of urban planning and construction; we shall make a 
uniform planning on various elements of gardens to promote harmony of gardens, and also promote the harmony between gardens and urban landscape from the perspective of urban planning. Then, in ecological landscape, we shall firstly determine geographical characteristics, and then reasonably arrange landscape architecture to realize consistency of partial landscape architecture and overall urban landscape. In urban planning, we shall not only make planning and design on urban landscape, but also blend cultural characteristics to show urban culture. As a part of urban planning, the ecological landscape design not only shall show beautiful scenery and exert the function of recreation and entertainment, but also shall show cultural deposits. Then, as for design of urban landscape architecture, we shall select plants with cultural deposits as much as possible. From ancient times, Chinese garden also endows various kinds of plants with specific connotation. Those plants endowed with culture color and become important role in landscape planting design and also become the specific cultural scenery in garden space. The cultural deposits contained in plants are all shown in time sequence; what those plants show in green landscape is a historic evolution.

\section{Strategic thoughts on ecological landscape design in landscape planning}

With people's higher pursuit of spiritual life, in order to keep natural and harmonious environment in garden planning and bring people a sense of relaxation, we shall carry out ecological landscape design from strategic perspective so as to compensate for the damage of ecological environment caused by urban industrial development. As for shaping of ecological landscape, we shall utilize green materials and adopt green design technology to meet urban residents' living demand.

(I) To make use of ecological theory to create ecological community in landscape planning

In urban landscape planning, the architecture is the main vein. In terms of formulating of architecture design and construction scheme, except for architecture design from perspective of landscape architecture, we shall also make spatial planning from ecological perspective to avoid the damage on surrounding ecological environment caused due to construction of garden architecture. As for architecture design and construction in gardens, while we establish green concept, use local materials, and make full use of local resources, we shall also consider natural benefits and social benefits that the garden architecture can exert. Based on the principle of following natural principles, as for original natural area in gardens, we shall keep their original features and cause no damage. Instead of damaging green landscape due to construction of garden architecture, we shall make reasonable use of natural condition of gardens to avoid the situation that the ecological system changes due to change of soil texture. The garden architecture is a fixed part of landscape planning, and it is also a part with rich landscape culture features; it will be injected with fashionable elements due to influence of social culture; however, it is inevitable that the garden architecture will continue the intercommunity of Chinese traditional culture to realize inheritance of garden architecture design from ancient times to the present. The architecture is cold and stiff; in order to make it become more vigorous, we shall rely on local water source and various kinds of plants to create humanized nature and keep original natural ecological features for the sake of promoting sustainable and stable development of ecological community.

(II) To select and use renewable resources in landscape planning as much as possible

According to the concept of suitable development of urban ecological environment, we shall select low-carbon lifestyle and carry out design on urban landscape from the perspective of green environmental protection so as to better conform to requirements of ecological environment landscape. In landscape planning, the design and construction of green landscape will consume large quantity of resources; in order to improve economic benefits and social benefits of ecological landscape, we shall use renewable resources and improve reusing efficiency. For example, the lighting design of landscape architecture, including matching facilities and daily use, will consume large quantity of resources. We can make full use of renewable resources and pay attention to green design of landscape architecture, which not only can improve the using efficiency of garden resources, but also can realize diversified utilization of resources. According to local natural conditions, we can utilize corresponding design means to select natural light and natural wind, or 
suitably select solar energy or wind energy to reduce consumption cost of resources. Meanwhile, as for use of renewable resources, we can also consider visual landscape and ecological benefits to reasonably design landscape and efficiently utilize renewable resources so as to meet natural ecological laws.

(III) To pay attention to creative level and promote diversification of landscape architecture in landscape planning

The ecological landscape design in landscape planning shall bring people comfortable feeling, and also have the function of promotion sublimation of people's aesthetic psychology. If we create the beauty of artistic conception according to Chinese traditional aesthetics, we can make landscape architecture full of unique charm. Therefore, we shall make diversified design on landscape architecture to form a spatial structure of natural ecological system. Chinese culture endows various kinds of plants with symbolic meaning; in terms of ecological landscape design, while keeping the features of natural environment, we shall also carry out artificial environment design. For example, the four kinds of plants (plum blossoms, orchid, bamboo and chrysanthemum) are called "four gentlemen"; in ecological landscape design, we can make a reasonable matching between those plants and landscape architecture to show cultural smell from natural environment. By use of features of plants, we can create garden environment full of artistic conception, which not only can realize good visual effect, but also can maintain stability of natural ecological community.

\section{Conclusion}

To sum up, the ecological landscape design in landscape planning shall start from the perspective of green ecological environment to make gardens exert the function of adjusting urban ecology and improve living environment. With the change of concepts of landscape design, there shall be innovative thoughts on ecological landscape design according to features of social development to inherit artistic design and endow individual color so as to conform to aesthetic renewable of modern society. We shall establish landscape design on the basis of protecting natural ecological environment to protect original ecological system, develop green landscape, realize harmony between urban gardens and urban planning landscape, and show cultural conception to form unique charm.

\section{Bibliography:}

[1] Qu Qiang: Discussion on Ground Cover Plants and Their Application in Gardens [J], Management and Technology of Small and Medium-sized Enterprises (periodical I), 2009 (08).

[2] Chen Rong: Discussion on Contemporary Urban Landscape Architecture Green Design [J], Urban Construction Theory Research (electronic edition), 2012 (27).

[3] Mang Hong, Mang Kunlun: Principles of Aesthetics for Urban Landscape Plant Configuration Design [J], Heilongjiang Science and Technology Information, 2010 (12).

[4] Hu Wenfang, Tan Lihua, Li Yanbo: Development Trend and Change of Contemporary Botanical Garden Design [C], Botanical Society of China Botanical Garden Branch Editorial Committee, collected papers of the $3^{\text {rd }}$ Global Botanic Garden Congress, Beijing: Chinese Forestry Publishing House, 2007.

[5] Liu Jing: Discussion on Thoughts of Contemporary Urban Landscape Green Design [J], Guide of Sci-tech Magazine, 2013 (27).

[6] Su Dexiang: Road of Ecological Design - Ecological Design Practice of One Team [M], Beijing: China Architecture \& Building Press, 2009.

[7] Gao Junxiao, Wang Xiufeng: Discussion on Relationship between Urban Aesthetics and Urban Garden Design [J], Xiandai Horticulture, 2013 (04).

[8] Zhou Jian: Current Situation and Development Trend of Modern Urban Landscape Design [J], Journal of Anhui Agricultural Sciences, 2012 (11).

[9] Huang Weiyong: Contemporary Urban Gardening Green Design[J], Journal of Anhui Agricultural Sciences, 2011.39 (31). 